\title{
Prophylactic Surgery and Extended Oncologic Radicality in Gastric and Colorectal Hereditary Cancer Syndromes
}

\author{
Holger Eduard Vogelsang \\ Department of General, Visceral, Thoracic and Endocrine Surgery, Klinikum Garmisch-Partenkirchen, \\ Teaching Hospital, Ludwig Maximilian University Munich, Garmisch-Partenkirchen, Germany
}

Keywords

Prophylactic surgery · Colorectal cancer - Gastric cancer . Hereditary

\section{Abstract}

Prophylactic surgery for high-penetrance hereditary gastric and colorectal cancer can be a primary prophylaxis of cancer and a secondary oncologic prevention. As early cancer is often detected in the resected organ, there has been no prophylaxis of cancer but cancer treatment. Extended oncological radicality with removal of the complete organ is a tertiary prevention as metachronous cancer is avoided. The indication for prophylactic surgery or extended oncological radicality is presented regarding hereditary and familial gastric and colorectal cancer. Hereditary diffuse type gastric cancer (E-cadherin mutation) and familial adenomatous polyposis coli (APC or MYH mutation) are well-accepted indications for prophylactic surgery with a variety of considerations regarding phenotype, genotype, associated diseases, age, timing, extent, and technique of surgery. Not so much prophylactic surgery as extended oncologic radicality can be considered in familial gastric and colorectal cancer as well as Lynch or hereditary nonpolyposis colorectal cancer syndrome (HNPCC). Clinical, molecular, and technical progress leads to less invasive and risk-adapted surgical and nonsurgical interventions, expanding the variety of individualized treatment options.

(c) 2019 S. Karger AG, Basel

\section{Introduction}

Cancer prevention is a rather intellectual approach to oncology. The majority of hereditary cancer syndromes identified so far are relatively rare and represent a small subgroup amongst the far more dominating population with so-called "sporadic cancer." An autosomal dominant hereditary pattern is often associated with a strongly positive family history, but this is either frequently missed or not known due to family fragmentation and patchwork situations. Identifying hereditary cancer patients and families is a tedious job to do for specialized clinicians, pathologists, geneticists, and family physicians. Success rates when identifying patients, persons, and families at risk are still far under theoretical limits. Penetrance of manifest tumor diseases and effective strategies for nonsurgical primary tumor prevention or early tumor detection influence the lifetime risk of a tumor disease with deadly outcome. Our increasing knowledge about new genes, gene interaction, and polygenic associations as well as phenotype-genotype correlations and modifying factors for lifetime tumor risk offers opportunities for risk stratification modulating therapeutic interventions, such as prophylactic surgical strategies. Once a patient presents with a tumor disease, partial organ preservation might be risky because of synchronous occult or metachronous tumor manifestation. Finally, the overall risk reduction, the possibility of compensating organ loss, and the risk of surgical intervention influence decisions for prophylactic surgery or extended oncologic radicality.

\section{KARGER}

(C) 2019 S. Karger AG, Basel
PD Dr. med. Holger Eduard Vogelsang 


\section{Terminology of Prevention and Prophylaxis in Epidemiology and Oncology}

Prevention is a well-defined term in epidemiology in general and oncology in particular. There is primary, secondary, and tertiary prevention. When referring to oncology, primary prevention is supposed to avoid the development of preneoplastic and neoplastic changes. Secondary prevention is defined as detection of preneoplastic or early subclinical cancer. Tertiary prevention is meant to be either preventing or controlling symptoms, morbidity of cancer or cancer therapy as well as preventing recurrence or second primary cancer [1]. From this point of view, prophylactic surgery of organs with preneoplastic changes is supposed to be a secondary prevention, although most of the literature refers to it as a "primary prophylaxis" of the tumor, which is true as long as no early cancers are present by the time of prophylactic surgery [2]. The NCI dictionary of cancer terms lists "prophylactic surgery" but not "preventive surgery" for removing organs before tumor development of patients at risk [3]. Therefore, we consistently use the term "prophylactic surgery" and avoid "preventive surgery."

\section{Intention of Prophylactic Surgery}

Prophylactic surgery wants to prevent tumor disease in order to avoid tumor-induced reduction of survival or life expectancy. This is the most important goal. Nevertheless, there is always a certain risk of treating early cancer or locally advanced undetected or overlooked tumor disease. By offering prophylactic surgery, we also want to avoid multimodal oncologic treatment and even more radical surgical procedures. By doing so, quality of life is most likely preserved much better than by treating obvious oncologic disease. Quality of life is the second goal just after tumor prevention. It is a matter of debate whether quality of life is better before or after prophylactic surgery. Basically, one should assume that quality of life is better with the organ at risk in situ than following organ resection. This is not generally true as the impact of the impending cancer risk affects the overall quality of life negatively even if the organ function is still preserved, and special organ-related items are rated positive before and negative after prophylactic surgery [4]. Prophylactic surgery is often classified by persons at risk as an important step forward into a risk-reduced life.

Prophylactic surgery is offered to proven germline mutation carriers with a high risk of tumor manifestation. Without germline mutation, a clear phenotype of cancer predisposition (i.e., adenomatous polyposis) also triggers indication for prophylactic surgery. Finally, the most difficult indication is a strong family history without a prov- en germline mutation and missing a preneoplastic phenotype as in familial gastric cancer. Even suggesting an autosomal dominant inheritance pattern, there is at least a $50 \%$ chance to perform prophylactic surgery on a nonmutation carrier. Nevertheless, some prophylactic gastrectomies have been performed in high-penetrance families before detecting E-cadherin germline mutations. Persons retrospectively identified as non-mutation carriers in families with a proven E-cadherin germline mutation reported a benefit of the operation as the subjective cancer risk burden had been reduced substantially. Therefore, the request for potential prophylactic surgery in high-risk families without a germline mutation is accompanied by intensive clinical, genetic, and psychological counseling and a rather cautious indication.

\section{Prophylactic Surgery and Extended Surgical Radicality in Hereditary Cancer Syndromes}

Prophylactic surgery is meant to prevent development of tumor. There might be a residual risk for metachronous tumor development after prophylactic surgery, which should be rather low due to, e.g., minimal residual organ mucosa or dystopic organ tissue. A precondition of prophylactic surgery in its original meaning is absence of tumor manifestation in the organ resected. Postoperative histopathological evidence of tumor manifestation turns prophylactic surgery into therapeutic oncologic surgery with the patient's outcome depending on tumor stage and applied surgical radicality while performing the suggested prophylactic surgery. Evidence of more than early mucosal cancer is a defeat for the prophylactic strategy and should lead to a reassessment of surveillance and the decisionmaking algorithm. Therefore, prophylactic surgery has to take into account the risk of treating occult tumor disease in order to choose appropriate surgical radicality. Once a tumor manifestation has developed and staging shows a completely removable tumor disease with or without indication for neoadjuvant therapy, potentially curative surgery can be offered to the patient. Depending on tumor localization and additional preneoplastic pathology, the affected organ does not need to be removed completely. Having in mind metachronous tumor risk due to germline mutation, extended oncologic radicality can be considered to reduce the future tumor risk of the patient. Extended radicality together with a primary tumor operation refers to the epidemiological definition mentioned above as tertiary prevention [1], whereas others define extended radicality at the time of the primary operation as "secondary prophylaxis." Performing extended radicality by a second surgical intervention after primary tumor resection and following postoperative diagnosis of hereditary tumor disposition has been proposed to be called "tertiary prophylaxis" [2]. 


\section{Associated Tumors}

Whenever prophylactic surgery for a hereditary tumor disposition can be considered, it is important to check the person at risk for other associated tumor diseases. There is hardly any heritable tumor disposition syndrome which is not prone to develop other synchronous or metachronous tumors. There might be some age dependency for metachronous disease as in familial adenomatous polyposis coli (FAP) and other tumors, such as papillary, gastric or thyroid cancer, developing later than polyposis of the colorectum. But obviously, synchronous tumor disease of the breast and gastric cancer in hereditary diffuse type gastric cancer (HDGC) is possible.

\section{Prophylactic Surgery in Gastric Cancer}

Hereditary Diffuse Type Gastric Cancer (Tables 1, 2)

Germline mutation of the E-cadherin gene defines the genetic basis of the HDGC syndrome. The E-cadherin gene has been identified as being responsible for the oncologic pathway of sporadic diffuse type gastric cancer in the early nineties' coding for a cell adhesion molecule [5]. In 1998, germline mutations in E-cadherin were described in New Zealand Maori families with a high prevalence for diffuse type gastric cancer manifestation in young patients [6]. Additionally, an increased prevalence for lobular type breast cancer was reported [7]. An international collaborative group on hereditary gastric cancer collected data and integrated expert experience of various medical disciplines in order to report on guidelines $[8,9]$. Lifetime risk of gastric cancer is about 57 and $70 \%$ in female and male mutation carriers and $42 \%$ for lobular breast cancer in female mutation carriers. The mean manifestation age is about 40 years for gastric and 46 years for lobular breast cancer in non-gastric cancer families [10]. As cancer risk is solely associated with diffuse type gastric cancer, early cancer detection by endoscopy is supposed to be rather difficult, at least in countries with an overall low gastric cancer prevalence. Due to difficult endoscopic surveillance and overall bad prognosis of diffuse type gastric cancer, prophylactic gastrectomy is considered the first choice with genetically proven HDGC and surveillance endoscopy the second choice $[8,9,11]$. Complete pathological workup of stomachs from E-cadherin germline mutation carriers revealed preneoplastic lesions, in situ diffuse type carcinoma, and early gastric cancer mostly of the mucosal type. Furthermore, multiple primary tumors have been identified in germline mutation carriers. Both the occurrence of preneoplastic lesions and multiple primaries can be interpreted as proof of principle of the germline mutation-tumor sequence. So far, there is no genotype-phenotype correlation. There-
Table 1. Checklist "prophylactic gastrectomy” for HDGC

- Age, comorbidities

- Surveillance endoscopy negative (no evidence of tumor)

- Treatment of dystopic gastric mucosa islands

- Clinical/psychological/genetic/nutritional counseling

- Laparoscopic (conventional) surgery

- D1 lymphadenectomy

- Complete intraoperative gastric mucosa resection (endoscopy, pathology frozen section)

- Macroscopic absence of gastric cancer

- Pouch reconstruction

- Parenteral vitamin B12 supplementation

- Intermittent nutritional and expert counseling

- Breast cancer surveillance

- Family support

Table 2. Indication for prophylactic surgery and extended radicality in hereditary and familial gastric and colorectal cancer

\begin{tabular}{lll}
\hline & $\begin{array}{l}\text { Prophylactic } \\
\text { surgery }\end{array}$ & $\begin{array}{l}\text { Extended } \\
\text { radicality }\end{array}$ \\
\hline $\begin{array}{l}\text { Gastric cancer } \\
\text { HDGC }\end{array}$ & yes & yes \\
$\begin{array}{l}\text { Lynch-/HNPCC-associated } \\
\text { gastric cancer }\end{array}$ & no & yes \\
$\begin{array}{l}\text { Familial gastric cancer } \\
\text { Colorectal cancer }\end{array}$ & no & yes \\
$\begin{array}{l}\text { Polyposis coli } \\
\text { Lynch syndrome/HNPCC }\end{array}$ & yes & yes \\
Familial colorectal cancer type X & no & yes \\
\hline
\end{tabular}

fore, mutation type does not modulate cancer risk or age of onset and recommendations for surveillance or prophylactic gastrectomy.

Prophylactic gastrectomy should be offered to persons at risk after intensive genetic, clinical, and psychological counseling within a hereditary family cancer clinic setting. Important issues are changing nutritional habits, weight loss of at least $10 \%$, and lifelong parenteral vitamin B12 supplementation. As there is early risk for gastric cancer development, the intervention age is between 20 and 30 years for known mutation carriers depending on the patient's personal training or education schedule [9]. A risk-benefit simulation model calculates the optimal prophylactic gastrectomy age for women at 30 years and men at 39 years of age [12]. It is debatable whether the individual psychological burden due to obvious tumor risk is sufficiently included into the risk calculation. There is supposed to be some increased quality of life in persons at risk with a high psychological burden following prophylactic gastrectomy. Age also depends on the time of the detection of the germline mutation; therefore, prophylactic gastrectomy is also offered to germline muta- 
tion carriers in later ages, considering their risk profile regarding comorbidities. Most persons at risk start with an initial diagnostic program and short-term surveillance with annual endoscopy and breast assessment. As intermittent clinical counseling is offered, most persons at risk take the offer and repeatedly discuss various aspects of the surgical and endoscopic options. Family events very often trigger the decision for prophylactic gastrectomy, as fatal outcomes of gastric cancer or unexpectedly newly diagnosed gastric cancer cases move so far indecisive germline mutation carriers.

Surveillance endoscopy as well as preoperative workup endoscopy for prophylactic gastrectomy should be performed with sufficient sedation, enabling an optimal endoscopic precondition, good inflation to expand the gastric wall, and thorough visualization of all quadrants with appropriate endoscopic magnification and narrow band imaging. Biopsies should be taken randomly and out of suspicious areas. Endoscopy has to refer to esophageal or duodenal gastric mucosa islands. They need to be followed up with the surveillance program, surgically removed with the prophylactic surgical procedure, or endoscopically treated by mucosal ablation before (duodenal, esophageal) or after (esophageal) prophylactic gastrectomy. The endoscopist and pathologist should be well informed about the genetic background to induce adequate awareness. No evidence of cancer is the precondition for prophylactic gastrectomy. Macroscopic or microscopic detection of gastric cancer induces possible extended staging, and, in case of curative surgery, D2 lymphadenectomy and total gastrectomy are the treatments of choice. Treatment of gastric cancer is performed in oncologic centers with an interdisciplinary collaboration of at least gastroenterology, pathology, medical and radiation oncology as well as oncological surgery. Outcome of surgery in gastric cancer is related to single surgical, departmental, and hospital experience [13]. Obviously, prophylactic gastrectomy should be offered by experienced gastric cancer surgeons with an adequate departmental and hospital structure considering complication management by endoscopic, radiological, or intensive care intervention. As the results of early gastric cancer surgery are comparable with either open conventional or minimally invasive surgery, the latter is the surgical procedure of choice for prophylactic gastrectomy [14]. The D1 lymph nodes along the greater and lesser curvature are supposed to be the first draining compartment in case of incidental early gastric cancer. Therefore, D1 lymphadenectomy should be part of the prophylactic gastrectomy procedure [9].

After removal of the stomach, thorough macroscopic inspection of the stomach specimen is performed either by the surgeon or, better, by the pathologist. Suspicious areas should be investigated by frozen section. Confirma- tion of early gastric cancer during a prophylactic gastrectomy procedure induces conversion of prophylactic to curative surgery with the extension to D2 lymphadenectomy, which can be easily performed by minimally invasive surgery. In case of histopathological evidence of D1 lymph node tumor infiltration postoperatively, additional D2 lymphadenectomy should be offered to the patient by a second operation. In order to minimize gastric cancer risk by prophylactic gastrectomy, complete removal of the gastric mucosa should be confirmed either by intraoperative endoscopy and macroscopy or frozen section of the esophagogastric and gastroduodenal transition.

Reconstruction following gastrectomy is a crucial topic. As there is strong evidence for pouch reconstruction even in gastric cancer patients, prophylactic surgery should undoubtedly be accompanied by pouch reconstruction [15]. There are various options for pouch reconstruction with the Hunt-Lawrence-Rodino pouch being worldwide the most favorite one [16]. As total gastrectomy is accompanied by at least partial or complete lower esophageal sphincter resection, pouch reconstruction with an antireflux barrier could improve quality of life in the long run. Our own reconstruction of choice is the Siewert-Peiper pouch, an esophagojejunoplication together with a jejunal pouch by the Roux technique [17]. So far, we performed 8 prophylactic gastrectomies in 7 E-cadherin germline mutation carriers and 1 case of familial gastric cancer without identified mutation. All have been done laparoscopically, 3 with extra-abdominal and 5 with complete laparoscopic pouch formation. Follow-up duration is between 6 months and 10 years with excellent quality of life (Tables 1,2 ).

\section{Gastric Cancer and Lynch Syndrome}

Gastric cancer in Lynch syndrome is number 3 following colorectal and endometrial cancer. About 5\% of Lynch-associated tumors are gastric cancers. The average manifestation age is about $56-58$ years with a predominance of intestinal type gastric cancer [18]. There is no common recommendation for gastric cancer screening in hereditary nonpolyposis colorectal cancer (HNPCC) in general. The German recommendations offer endoscopic surveillance for germline mutation carriers starting at 35 years of age [19]. At least in families with gastric cancer cases, endoscopy should be offered to first-degree relatives 5-10 years earlier than the youngest gastric cancer patient in the pedigree. Obviously, due to a comparatively low gastric cancer incidence and, therefore, moderate lifetime risk, there is no indication for prophylactic gastrectomy in hereditary nonpolyposis colorectal cancer (HNPCC) with proven germline mutation in DNA mismatch repair genes. Nevertheless, gastric cancer may be multifocal initially, and metachronous risk is increased. 
Gastric cancer patients and their families should be screened not only for HDGC but also for Lynch syndrome. In any case of Lynch-associated gastric cancer, total gastrectomy rather than subtotal resection should be performed even if appropriate for the actual tumor manifestation (intestinal type, tumor stage, distal localization). Also, metachronous multifocal gastric cancer treated endoscopically might be an indication for total gastrectomy. All these scenarios are described by the term "extended oncologic radicality," performing either total instead of subtotal gastric resection or gastrectomy instead of repeated endoscopic resection.

\section{Familial Gastric Cancer}

Gastric cancer presents with a positive family history in first- or second-degree family members in about 15$25 \%$ with a decreasing prevalence in western countries. A strongly positive family history should induce genetic counseling in order to check for an inheritance pattern, candidate genes, or a multigene panel testing. Most cases will be mutation negative. In case of surgical treatment, there are potentially 2 options: subtotal and total gastrectomy. A positive family history should assume a higher risk for multifocal and metachronous gastric cancer. Therefore, total gastrectomy in principle could be an option of extended radicality considering age and comorbidities of the patient.

\section{Prophylactic Surgery in Colorectal Cancer}

\section{Polyposis Coli}

Whenever multiple polyps in the colon or rectum are detected, distribution, number, appearance, histology, and family history provide important information about the potential genetic background, tumor risk, and therapeutic options. This paper is not supposed to deal with the different types of polyposis diseases but the indication for prophylactic surgery. Adenomatous polyps, more than 100 in number, and a strong family history for colorectal cancer are very strong indicators for FAP caused by germline mutation in the APC gene with an autosomal dominant inheritance pattern. A missing family history can be suggestive for MYH mutation with an autosomal recessive inheritance pattern, a new mutation in the APC gene, a post-germline mosaic pattern, or a so far unknown mutation [20]. In all patients with multiple adenomatous polyps, the potential of endoscopic surveillance, patient's compliance, comorbidities, and the degree of intraepithelial neoplasia or carcinoma development are decisive for potential prophylactic surgery or extended surgical radicality in case of single cancer development. This is true for persons at risk with detected germline mutations but particularly for persons at risk
Table 3. Checklist "prophylactic colorectal resection" in polyposis

- Polyposis phenotype and time course of progress,

- Age, comorbidities

- Extracolorectal tumor check

- APC genotype

- Desmoid family history, presence of desmoid tumors

- Family planning for male and female carriers

- Clinical/psychological/genetic/nutritional counseling

- Surveillance compliance

- Colectomy with ileorectal anastomosis

- Coloproctectomy with ileal pouch-anal anastomosis

(handsewn, double-stapling, TaTME) with/without ileostomy

- Surveillance (endoscopic, general)

- Chemoprevention

- Secondary surgery

- Family support

without identified mutational background. The extent of prophylactic surgery depends on colorectal morphology, the dynamic of polyp expansion, options of chemoprevention, and family planning particularly in young women. There is a wide range of individual or standardized surgical options: segmental resection, standard oncologic resection, subtotal colon resection, total colectomy, and subtotal or total colorectal resection (proctocolectomy) with or without ileoanal pouch reconstruction.

\section{APC Gene Germline Mutation or}

FAP/MYH-Associated Polyposis (Tables 2, 3)

FAP is the classic hereditary tumor disposition disease mostly due to germline mutation in the APC gene. Three clinical variants determine different approaches: early childhood manifestation, typical manifestation between 15 and 25 years, and attenuated manifestation with later or milder onset. Besides the other aspects mentioned, morphologic manifestation and clinical course including symptoms are the leading aspects of therapeutic decisionmaking. Interestingly, the type of mutation can give additional relevant hints on the time, radicality, and even approach of appropriate prophylactic surgery. There is some genotype-phenotype correlation defining the extent of polyposis and the probability of developing desmoid tumors, partly due to the surgical trauma. There are mutations (codon 0-178, 312-412) with a rather late onset of polyposis and good survival compared to early onset and worse survival (codon 1,249-1,549). Some mutations (codon 1,250-1,464) bear a high risk for adenoma progression in the rectum, and mutations beyond codon 1,550 bear a high risk of desmoid development following surgery ( $85 \%$ with surgery vs. $10 \%$ without surgery) [21-26].

Whenever possible, prophylactic surgery should be offered as a laparoscopic minimally invasive procedure. There are hardly any contraindications. Even previous 
complicated surgery does not necessarily lead to primary conventional open surgery as long as laparoscopic exploration offers the chance for sufficient and safe adhesiolysis and access to relevant structures, such as the mesentery and pelvic organs. There is a relevant probability of single or multifocal cancer depending on the age, the clinical variant, and the time course of the polyposis disease. Therefore, oncologic resection with systematic lymphadenectomy should be performed in case of suspicion of early cancer. Mild polyposis, small number of rectal adenomas $(<20)$ and small size $(\leq 9 \mathrm{~mm})$, and high risk for desmoids (except mutations $>$ codon 1,444) as well as planned pregnancies favor ileorectal anastomosis. With an exceptionally high risk for desmoids, some authors would even accept adenomas up to a size of $20 \mathrm{~mm}$ [2]. Twenty or more rectal adenomas, getting at least larger than $9 \mathrm{~mm}$, high-grade intraepithelial neoplasia, and typical FAP manifestation ( $>1,000$ polyps) favor proctocolectomy and ileo-pouch-anal anastomosis $[2,27,28]$. Particularly the different impact of ileorectal anastomosis and ileoanal pouch formation has to be considered. Ileorectal anastomosis is associated with a lower complication rate, preserved sphincter function, and intact pelvic floor and nerve anatomy. There is a necessity for intensive polyp surveillance on average every 6 and sometimes every 3 months with increased cancer risk and often secondary surgery with pouch-anal anastomosis. A precondition is optimal patient's compliance. Depending on the initial selection criteria, $30-50 \%$ of ileorectal anastomoses have to be converted to pouch-anal anastomoses or terminal ileostomy because of increasing cancer risk (progressive polyposis, intraepithelial neoplasia, or early cancer). Risk for secondary rectal loss is increased 4 times with a mutation between codon 1,250 and 1,450 and 2 times with the initial operation before the age of 25 years $[29,30]$. Ileo-pouch-anal anastomosis reduces cancer risk substantially, with increased risk for operative complications $(20 \%$ [5-30\%]), ileal pouch failure $(3.8 \%$ [3.8$10 \%]$ ), and impaired sphincter function $(20-60 \%)$ [26, $31,32]$. Either total mesorectal excision or a partial mesorectal sparing technique is applied. The latter is better for nerve preservation, is probably desmoid inducing and insufficiently radical in case of occult rectal cancer [28]. Additionally, high ligation of the ileocolic artery should be avoided initially, at least if oncological lymph node aspects allow a preservation, because formation of the pouch might favor transection of an ileal artery to achieve enough mesenteric length for a tension-free pouch-anal anastomosis. J- or S-pouch is favored worldwide with a tendency towards 1-step procedures without deviating ileostoma. Ileostoma might create some tension to the pouch-anal anastomosis. Möslein [28] proposed a "ghost ileostoma," a prepared ileal loop fixed to the abdominal wall, which can be converted to an ileostomy in case of a leakage [33]. As a handsewn anastomosis compared to a stapled anastomosis increases the incontinence rate for liquid stool (29.4 vs. $22.1 \%)$ and stool incontinence at night ( 29.8 vs. $16.8 \%)$, which is negatively influenced by mucosectomy as well, there is a tendency towards stapled anastomosis without transanal mucosectomy, accepting a slightly increased cancer risk due to residual mucosa close to the pouch-anal anastomosis [31]. There is some experience with transanal total mesorectal excision (TaTME) for pouch-anal anastomosis with no larger series on polyposis patients and their functional results so far. Nevertheless, functional results, adenoma incidence, and cancer risk should be similar to transanal mucosectomy and handsewn anastomosis $[34,35]$. Overall, functional results demonstrate better results in younger patients with an age-dependent incontinence rate between 20 and $60 \%$ but a general satisfaction rate in all age groups of at least 90-95\% [32].

The phenotypic rectal adenoma load determines the possibility of rectal preservation; the genotype modulates the operative procedure, and the attenuated manifestation allows later surgical intervention with a lower extent of resection. Chemoprevention increases cardiovascular risk and does not avoid the necessity for secondary surgery in case of rectal polyposis progression.

The same is true for polyposis and familial colorectal cancer caused by rare germline mutations in POLD1, POLE, NTHL1, and MSH3 [20, 36]. MYH-associated polyposis is an autosomal recessive inherited polyposis closely resembling APC-associated disease. The pattern is often similar to the attenuated FAP, predominantly rightsided with a later manifestation or onset of symptoms. Morphology and course of the disease primarily influence surgical decisions as in FAP (Tables 2, 3).

\section{Hereditary Nonpolyposis Colorectal Cancer}

\section{Lynch Syndrome with and HNPCC without Mismatch} Repair Gene Mutations (Tables 2, 4)

Lynch syndrome (the term HNPCC is often synonymously used) is defined as HNPCC with microsatellite instable tumors and germline mutation of the mismatch repair genes MLH1, MSH2, PMS2, MSH6, and EPCAM. Contrary to FAP, there is a predominant phenotypic pattern of single or few polyps, mainly adenomas, with a preference for the right colon. Although there is a high overall penetrance with a lifetime tumor risk of about 70 $80 \%$, the predominant tumor is colorectal cancer with an option of surveillance by annual colonoscopy and endoscopic adenoma resection. Colorectal cancer is followed by endometrial, gastric, duodenal, ovary, and urothelial cancer (mainly pelvis and ureter, partly bladder). There is some genotype-phenotype correlation as MSH6 muta- 
Table 4. Checklist "extended radicality" in Lynch-/HNPCC-associated colorectal cancer

- Localization of primary tumor and number of adenomas

- Age and comorbidities

- Genotype: type of mutated gene

- Surveillance compliance

- Previous extracolorectal associated tumors

- Extracolorectal tumor check

- Prophylactic hysterectomy and oophorectomy

- Subtotal/total colectomy/coloproctectomy

- Ileorectal anastomosis, ileo-pouch-anal anastomosis

- Intensive postoperative surveillance

- Family support

tions show an overall reduced lifetime risk for cancer with a later age of onset. Patients experience a considerable synchronous and metachronous cancer risk even with good compliance and annual surveillance colonoscopy of some $10-15 \%$ within 10 years following the first colorectal cancer [37].

We use the term HNPCC for patients and persons at risk with a positive family history fulfilling the Amsterdam criteria, a tumor genotype with microsatellite instability but without detection of germline mutations. Decisions regarding oncologic, prophylactic, or extended surgery are the same as in Lynch syndrome with a given germline mutation. Persons at risk can be identified via phenotype only when presenting with adenomas or carcinomas with HNPCC-related clinical and genotypic features.

Prophylactic surgery in Lynch syndrome mutation carriers is not supposed to be a serious option from a European point of view. There is some discussion in the US whether prophylactic surgery should be offered to persons at risk with a high psychological burden, aggressive familial phenotype, and early-onset manifestation. Once a patient experiences colorectal tumor manifestation, there should be a discussion about extended surgery [2] For left- or right-sided colon cancer, colectomy with ileorectal anastomosis can be discussed. Although functional aspects following total colectomy are worse than following right- or left-sided colectomy, quality of life is rated the same by Lynch patients [4]. Otherwise, the option of prophylactic extended radicality can be challenged. It is well known that Lynch-associated colorectal cancer has a stage-related better prognosis than sporadic cancer, although the adenoma-carcinoma sequence is shorter. Annual surveillance colonoscopy with good patient's compliance leads to an early diagnosis of colorectal cancer. These cancers diagnosed between 2 surveillance endoscopies are called interval cancer [38]. Modern endoscopy can define risk potentials of early colorectal cancer. Anatomic-topographic studies of lymph node involvement of colorectal cancer related to the $\mathrm{T}$ category demonstrate that the region of involved lymph nodes and their localization for early colorectal cancer are close to the tumorbearing segment [39]. In case of proven interval cancer with a low-risk profile, limited segmental resection with central vessel-sparing lymphadenectomy might be sufficiently radical and effective regarding the actual tumor disease. There might also be a future perspective for a renaissance of sentinel lymph node detection in this subgroup of Lynch patients. Fifteen percent of initial colorectal cancer cases in Lynch patients are located in the rectum. Metachronous risk of colon cancer after proctectomy is about $15-25 \%$ within 10 years and even more than $50 \%$ after 30 years [40]. Following extended radicality for rectal cancer with total colectomy beforehand, there is no reconstructive option for the colon for rectal cancer patients. Ileal pouch-anal anastomosis or terminal continent or incontinent ileostomy are the only options. Most surgeons even in favor of prophylactic surgery in Lynch syndrome would not advocate extended radicality by ileo-pouch-anal anastomosis for rectal cancer unless it is necessary for other reasons [2].

Prophylactic surgery for female Lynch patients should be considered regarding hysterectomy and oophorectomy at least after finishing family planning. The overall risk for gynecologic tumors is slightly lower than initially calculated with high-risk families. As risk for ovarian cancer is much lower than for endometrial cancer and oophorectomy before menopause is burdensome, prophylactic resection of the ovaries is advised rather after than before menopause. In case of primary colorectal cancer with Lynch syndrome, prophylactic hysterectomy and oophorectomy should be considered and discussed with the patient $[37,41]$ (Tables 2, 4).

\section{Familial Colorectal Cancer Type X}

Amsterdam-positive family history and exclusion of microsatellite instability tumors as well as negative mutation analysis for other relevant germline mutations (multigene panel analysis) are defined as familial colorectal cancer type $\mathrm{X}$. The average manifestation age is somewhat higher in these families with a lower frequency of extracolorectal tumor diseases. Metachronous colorectal cancer risk is less, as is the incidence of colorectal adenomas, compared to Lynch syndrome and HNPCC [42-44]. Therefore, cancer risk has to be calculated on a much lower level with no recommendation so far regarding extended radicality in patients with tumor manifestation. As there is no identification of germline mutation and no other individual phenotypic identification of persons at risk, no prophylactic surgery in case of preneoplastic changes can be offered. 


\section{Conclusion}

Indication for prophylactic surgery or extended radicality in hereditary gastrointestinal tumor disposition is a matter of risk calculation and secondary therapeutic options. Surveillance efficacy, chemoprevention, psychological burden, quality of life, and disease-associated secondary risks are important modulating factors. Increasing knowledge about the genotype and phenotype exerts influence on the decision-making process. Less invasive and risk-adapted surgical and nonsurgical interventions expand the variety of individualized options. Study inclu- sion and caretaking in a hereditary cancer center with a multiprofessional board is a precondition for generating and offering optimal advancing knowledge.

\section{Disclosure Statement}

The author has no conflicts of interest to declare.

\section{Funding Sources}

There are no funding sources.

\section{References}

1 Blackburn EH, Tlsty TD, Lippman SM. Unprecedented opportunities and promise for cancer prevention research. Cancer Prev Res (Phila). 2010 Apr;3(4):394-402.

2 Church JM. Controversies in the surgery of patients with familial adenomatous polyposis and Lynch syndrome. Fam Cancer. 2016 Jul; 15(3):447-51.

3 NIH National Cancer Institute. NCI dictionary of cancer terms [Internet]. Available from: https://www.cancer.gov/publications/dictionaries/cancer-terms

4 Haanstra JF, de Vos Tot Nederveen Cappel WH, Gopie JP, Vecht J, Vanhoutvin SA, Cats A, et al. Quality of life after surgery for colon cancer in patients with Lynch syndrome: partial versus subtotal colectomy. Dis Colon Rectum. 2012 Jun;55(6):653-9.

5 Becker KF, Atkinson MJ, Reich U, Becker I, Nekarda H, Siewert JR, et al. E-cadherin gene mutations provide clues to diffuse type gastric carcinomas. Cancer Res. 1994 Jul;54(14): 3845-52.

6 Guilford P, Hopkins J, Harraway J, McLeod M, McLeod N, Harawira P, et al. E-cadherin germline mutations in familial gastric cancer. Nature. 1998 Mar;392(6674):402-5.

7 Keller G, Vogelsang H, Becker I, Hutter J, Ott $\mathrm{K}$, Candidus S, et al. Diffuse type gastric and lobular breast carcinoma in a familial gastric cancer patient with an E-cadherin germline mutation. Am J Pathol. 1999 Aug;155(2):33742 .

8 Caldas C, Carneiro F, Lynch HT, Yokota J, Wiesner GL, Powell SM, et al. Familial gastric cancer: overview and guidelines for management. J Med Genet. 1999 Dec;36(12):873-80.

9 van der Post RS, Vogelaar IP, Carneiro F, Guilford P, Huntsman D, Hoogerbrugge N, et al. Hereditary diffuse gastric cancer: updated clinical guidelines with an emphasis on germline $\mathrm{CDH} 1$ mutation carriers. J Med Genet. 2015 Jun;52(6):361-74.

10 Corso G, Intra M, Trentin C, Veronesi P, Galimberti V. CDH1 germline mutations and hereditary lobular breast cancer. Fam Cancer. 2016 Apr;15(2):215-9.
11 Fitzgerald RC, Hardwick R, Huntsman D, Carneiro F, Guilford P, Blair V, et al.; International Gastric Cancer Linkage Consortium. Hereditary diffuse gastric cancer: updated consensus guidelines for clinical management and directions for future research. J Med Genet. $2010 \mathrm{Jul} ; 47(7): 436-44$.

12 Laszkowska M, Silver E, Schrope B, Kastrinos F, Wang TC, Hur C. Optimal Timing of Total Gastrectomy to Prevent Diffuse Gastric Cancer in Individuals with Pathogenic Variants in CDH1. Clin Gastroenterol Hepatol. 2019 Jun;S1542-3565(19)30647-0.

13 Grundmann RT, Hölscher AH, Bembenek A, Bollschweiler E, Drognitz O, Feuerbach S, et al. [Diagnosis of and therapy for gastric cancer-work-flow]. Zentralbl Chir. 2009 Aug; 134(4):362-74.

14 Berlth F, Yang HK. Minimal-invasive gastrectomy: what the west can learn from the east? Updates Surg. 2018 Jun;70(2):181-7.

15 Gertler R, Rosenberg R, Feith M, Schuster T, Friess H. Pouch vs. no pouch following total gastrectomy: meta-analysis and systematic review. Am J Gastroenterol. 2009 Nov; 104(11): 2838-51.

16 El Halabi HM, Lawrence W Jr. Clinical results of various reconstructions employed after total gastrectomy. J Surg Oncol. 2008 Feb;97(2): 186-92.

17 Siewert JR, Peiper HJ, Jennewein HM, Waldeck F. [Esophago-jejunoplication. Technic of anastomosis for the prevention of reflux following total gastrectomy]. Chirurg. 1973 Mar;44(3):115-20.

18 Vogelsang H, Ott K, Mehler J, Keller G, Siewert JR. Prophylaktische Chirurgie des Magens. Chirurg. 2005 Dec;76(12):1115-24.

19 Steinke V, Engel C, Büttner R, Schackert HK, Schmiegel WH, Propping P. Erblicher Darmkrebs ohne Polyposis. Dtsch Arztebl Int. 2013; 110(3):32-8.

20 Short E, Sampson J. The role of inherited genetic variants in colorectal polyposis syndromes. Adv Genet. 2019;103:183-217.

21 Nagase H, Miyoshi Y, Horii A, Aoki T, Ogawa $\mathrm{M}$, Utsunomiya J, et al. Correlation between the location of germ-line mutations in the APC gene and the number of colorectal polyps in familial adenomatous polyposis patients. Cancer Res. 1992 Jul;52(14):4055-7.
22 Vasen HF, van der Luijt RB, Slors JF, Buskens E, de Ruiter P, Baeten CG, et al. Molecular genetic tests as a guide to surgical management of familial adenomatous polyposis. Lancet. 1996 Aug;348(9025):433-5.

23 Marabelli M, Molinaro V, Abou Khouzam R, Berrino E, Panero M, Balsamo A, et al. Colorectal Adenomatous Polyposis: Heterogeneity of Susceptibility Gene Mutations and Phenotypes in a Cohort of Italian Patients. Genet Test Mol Biomarkers. 2016 Dec;20(12): 777-85.

24 Newton KF, Mallinson EK, Bowen J, Lalloo F, Clancy T, Hill J, et al. Genotype-phenotype correlation in colorectal polyposis. Clin Genet. 2012 Jun;81(6):521-31.

25 Nieuwenhuis $\mathrm{MH}$, Lefevre JH, Bülow S, Järvinen H, Bertario L, Kernéis S, et al. Family history, surgery, and APC mutation are risk factors for desmoid tumors in familial adenomatous polyposis: an international cohort study. Dis Colon Rectum. 2011 Oct;54(10): 1229-34.

26 Caspari R, Olschwang S, Friedl W, Mandl M, Boisson C, Böker T, et al. Familial adenomatous polyposis: desmoid tumours and lack of ophthalmic lesions (CHRPE) associated with APC mutations beyond codon 1444. Hum Mol Genet. 1995 Mar;4(3):337-40.

27 Vasen HF, Möslein G, Alonso A, Aretz S, Bernstein I, Bertario L, et al. Guidelines for the clinical management of familial adenomatous polyposis (FAP). Gut. 2008 May;57(5):70413.

28 Möslein G. Surgical considerations in FAPrelated pouch surgery: could we do better? Fam Cancer. 2016 Jul;15(3):457-66.

29 Koskenvuo L, Renkonen-Sinisalo L, Järvinen HJ, Lepistö A. Risk of cancer and secondary proctectomy after colectomy and ileorectal anastomosis in familial adenomatous polyposis. Int J Colorectal Dis. 2014 Feb;29(2):22530.

30 Sinha A, Tekkis PP, Rashid S, Phillips RK, Clark SK. Risk factors for secondary proctectomy in patients with familial adenomatous polyposis. Br J Surg. 2010 Nov;97(11):1710-5. 
31 Lovegrove RE, Constantinides VA, Heriot AG, Athanasiou T, Darzi A, Remzi FH, et al. A comparison of hand-sewn versus stapled ileal pouch anal anastomosis (IPAA) following proctocolectomy: a meta-analysis of $4183 \mathrm{pa}$ tients. Ann Surg. 2006 Jul;244(1):18-26.

32 Erkek AB, Church JM, Remzi FH. Age-related analysis of functional outcome and quality of life after restorative proctocolectomy and ileal pouch-anal anastomosis for familial adenomatous polyposis. J Gastroenterol Hepatol. 2007 May;22(5):710-4.

33 Warrier SK, Kalady MF. Familial adenomatous polyposis: challenges and pitfalls of surgical treatment. Clin Colon Rectal Surg. 2012 Jun;25(2):83-9.

34 de Lacy FB, Keller DS, Martin-Perez B, Emile $\mathrm{SH}$, Chand M, Spinelli A, et al. The current state of the transanal approach to the ileal pouch-anal anastomosis. Surg Endosc. 2019 May;33(5):1368-75.

35 Zaghiyan K, Warusavitarne J, Spinelli A Chandrasinghe P, Di Candido F, Fleshner P. Technical variations and feasibility of transanal ileal pouch-anal anastomosis for ulcerative colitis and inflammatory bowel disease unclassified across continents. Tech Coloproctol. 2018 Nov;22(11):867-73.
36 Valle L, Hernández-Illán E, Bellido F, Aiza G, Castillejo A, Castillejo MI, et al. New insights into POLE and POLD1 germline mutations in familial colorectal cancer and polyposis. Hum Mol Genet. 2014 Jul;23(13):3506-12.

37 Vasen HF, Blanco I, Aktan-Collan K, Gopie JP, Alonso A, Aretz S, et al.; Mallorca group. Revised guidelines for the clinical management of Lynch syndrome (HNPCC): recommendations by a group of European experts. Gut. 2013 Jun;62(6):812-23.

38 Engel C, Rahner N, Schulmann K, HolinskiFeder E, Goecke TO, Schackert HK, et al.; German HNPCC Consortium. Efficacy of annual colonoscopic surveillance in individuals with hereditary nonpolyposis colorectal cancer. Clin Gastroenterol Hepatol. 2010 Feb; 8(2):174-82.

39 Hida J, Okuno K, Yasutomi M, Yoshifuji T, Uchida $\mathrm{T}$, Tokoro $\mathrm{T}$, et al. Optimal ligation level of the primary feeding artery and bowel resection margin in colon cancer surgery: the influence of the site of the primary feeding artery. Dis Colon Rectum. 2005 Dec;48(12): 2232-7.
40 Kalady MF, Lipman J, McGannon E, Church JM. Risk of colonic neoplasia after proctectomy for rectal cancer in hereditary nonpolyposis colorectal cancer. Ann Surg. 2012 Jun; 255(6):1121-5.

41 Manchanda R, Menon U, Michaelson-Cohen R, Beller U, Jacobs I. Hereditary non-polyposis colorectal cancer or Lynch syndrome: the gynaecological perspective. Curr Opin Obstet Gynecol. 2009 Feb;21(1):31-8.

42 Mueller-Koch Y, Vogelsang H, Kopp R, Lohse P, Keller G, Aust D, et al. Hereditary non-polyposis colorectal cancer: clinical and molecular evidence for a new entity of hereditary colorectal cancer. Gut. 2005 Dec;54(12): 1733-40.

43 Lindor NM, Rabe K, Petersen GM, Haile R, Casey G, Baron J, et al. Lower cancer incidence in Amsterdam-I criteria families without mismatch repair deficiency: familial colorectal cancer type X. JAMA. 2005 Apr; 293(16):1979-85.

44 Hatfield E, Green JS, Woods MO, Warden G, Parfrey PS. Impact of colonoscopic screening in Familial Colorectal Cancer Type X. Mol Genet Genomic Med. 2018 Nov;6(6):102130. 